\title{
RESEÑAS
}




\section{LA ESCUELA Y LA FORMACION DE LECTORES}

Natalia Becerra

En la actualidad el problema de la formación de lectores es una preocupación de todos los niveles educativos en nuestros países latinoamericanos.

Esta obra presenta algunos aspectos alrededor de los cuales el lector podrá reflexionar con respecto a la problemática de la lectura. Así cada unidad temática presenta diversos aspectos de esta problemática y genera en el lector el interés por plantear sus propios puntos de vista.

Las unidades temáticas desarrolladas en siete diferentes textos, los cuales están apoyados por un audiovisual, son:

1.- Hacía una Nueva Pedagogía de la Lectura.

2.- Los primeros pasos en la formación del niño lector.

3.- La Biblioteca y la formación de lectores.

4.- Relaciones alumno-maestro en la formación de lectores.

5.- El placer de leer en un programa de lectura.

6.- La producción de textos en un programa de lectura.

7.- Los niños investigadores y la obra documental.

Los textos son resultado del Proyecto de investigación -acción Laboratorio de literatura Infantil y Lectura.

Cada texto se presenta de tal manera que quienes lean puedan a su vez construir nuevos conocimientos y desarrollar distintas actividades para promover la lectura en su medio.

1.-Hacia una Nueva Pedagogía de la lectura.

María Elvira Charria de Alonso y Ana González.

El desarrollo de una pedagogía activa en la escuela se ha convertido en el propósito de muchos maestros, quienes buscan hacer de la lectura el eje mismo del aprendizaje.

Para que la lectura sea el eje del aprendizaje es necesario crear situaciones dentro de la escuela y el aula, que reúnan las condiciones de lectura verdadera en función de la comunicación.

Pero cuáles son estas condiciones que debe crear la escuela?

Los aspectos tratados en esta unidad temática dan respuesta a este interrogante y por lo tanto sus desarrollos se centran en:

a) La concepción de la lectura como un acto complejo.

b) Los materiales de lectura.

c) El aprendizaje de la lectura.

d) Formulación y desarrollo de estrategias para promover la lectura. 
2.-Los primeros pasos en la formación del niño lector.

\section{María Josefa Charria de Gómez y Manía Elvira de Alonso}

Si partimos de que la lectura es un acto complejo, la formación del lector en todas las edades, también lo es.

Así pues que el papel de la escuela es fundamental en la iniciación y continuación de la formación del lector. Esta debe crear condiciones de contacto real del niño con el libro, con la palabra escrita.

Los autores plantean que las condiciones se dan cuando generan intereses en el niño de aprender a leer y es aquí básicamente donde se desarrollan las ideas de esta unidad temática.

La escuela debe generar en el niño el interés por aprender a leer, continuando la labor de la familia, en algunos casos, pero en muchos iniciándolo en sus primeros contactos.

El texto entonces desarrolla ideas alrededor de la maduréz para la lectura vs. el interés por la lectura, el interés por leer y aprendizaje lector, facilitación por parte del adulto del contacto del niño con el sistema escrito, la significación del texto escrito y la utilización de otros medios de expresión para comunicarse.

\section{3.-El placer de leer en un programa de lectura. Ana González y María Elvira de Alonso}

El porcentaje de adultos, jóvenes e incluso niños que viven la lectura como una a "tarea difícil y tediosa", es bastante alto en nuestro medio. La razón? El haber estado en una escuela donde los propios maestros no han construido el valor de la lectura como una actividad interesante y placentera, pues la pedagogía ha centrado su interés en que el lector sea un decodificador.

Con la anterior premisa, los autores desarrollan algunas ideas de como la escuela y el maestro podrían hacer que el niño tuviese una relación activa con el texto escrito, dándole oportunidades de tener contacto con textos que respondan a sus necesidades psicológicas y afectivas.

Para esto proponen empezar con la lectura de cuentos y poesía y gradualmente con la del texto documental.

4.-Relaciones alumno-maestro en la Formación de Lectores. Natalia Becerra Cano y María Elvira de Alonso.

En este texto las autoras desarrollan sus ideas alrededor de la importancia de la relación pedagógica alumno-maestro en la formación de lectores

En un primer momento se muestra como la historia de aprendizaje del maestro determina una relación específica con sus alumnos y como esta relación se refleja en las formas de trabajo, en la utilización del material escrito y en la evaluación de la lectura

En un segundo momento se trabajan ideas que señalan que tipo de relación pedagógica alumno-maestro, permite que en la escuela se formen lectores. Esta relación pedagógica está basada en la verdadera comunicación, donde las formas de trabajo en el aula, el uso de materiales escritos y las formas de evaluación de la lectura lleva a la formación de lectores autónomos, incluso a la formación de un maestro lector. 
5.-La producción de textos en un Programa de lectura. Ana González y María Elvira de Alonso

Las autoras de este texto desarrollan sus ideas alrededor del acto escritor, señalando que es tan complejo como la lectura y que está íntimamente relacionado con este último.

Primero muestran como la tarea de escribir en la escuela está desvinculada dc la lectura y se ha reducido a aprender la ortografía, algo de puntuación y a hacer una bonita caligrafía. Por lo tanto las formas de trabajo escolar llevan a que el niño copie modelos ya sean las letras aisladas (planas), el dictado del maestro o el texto escolar, sin que esto tenga un significado comunicativo para él. Así, enfocada la concepción de lo que es escribir y de lo que es aprender a escribir, el acto escritor pierde su función comunicativa y expresiva.

Las autoras muestran entonces, en esta unidad, como el niño aprende la lengua escrita cuando su aprendizaje se da en condiciones que le permiten entender el valor de la lengua escrita como "medio de comunicación", como "fuente de información"y como "una manera de evitar el olvido".

En este contexto entonces se muestra cual debe ser el papel de la escuela, del maestro y del adulto para que el niño sea escritor siendo lector.

\section{6.-Los niños investigadores y la obra Documental. Natalia Becerra Cano y María Elvira de Alonso}

Este libro presenta algunas ideas sobre como hacer de la obra documental en la escuela el eje central de la construcción de conocimiento sobre el mundo, por parte del niño. Así pues se plantea la función de las preguntas y los intereses de los niños como punto de partida en la actividad investigativa y el papel del libro documental en eta búsqueda.

Desarrollan las autoras, entonces, ideas alrededor del tipo de texto documental según permita al lector su cooperación activa en la construcción de su significado y hablan sobre el papel del maestro como facilitador de esta "apertura".

Por otro lado, presentan aspectos generales sobre como estructurar estrategias de lectura alrededor de la actividad investigativa en Ciencias y Ciencias Sociales.

7.-La Biblioteca y la Formación de Lectores.

María Josefa Charria de Gómez y María Elvira de Alonso

Las autoras en este texto señalan aspectos que hacen de la Biblioteca un recurso esencial en la formación de lectores.

En consecuencia, desarrollan ideas respondiendo a: quiénes utilizan la biblioteca, cuándo la utilizan y cómo, articulando su discurso a la relación biblioteca-aula escolarcomunidad. Así por ejemplo, al hablar de quiénes utilizan la biblioteca, hablan realmente de como hacer que los usuarios realmente la utilicen y la sientan suya.

También plantean desarrollos sobre las condiciones físicas y funcionales que hacen de la biblioteca un recurso en la formación de lectores. Así por ejemplo proponen algunas 
actividades de la biblioteca hacia la promoción de la lectura en la escuela y en la comunidad.

Esta investigación es financiada por el Ministerio de Educación Nacional, por PROCULTURA y el CERLALC. 